SANDIA REPORT

SAND95-2790 • UC-706

Unlimited Release

Printed December 1995
REr

FEB 14 ino

OSTI

\title{
Local Tetrahedron Modeling of Microelectronics Using the Finite-Volume Hybrid-Grid Technique
}

Douglas J. Riley, C. David Turner

\section{Prepared by}

Sandia National Laboratories

Albuquerque, New Mexico 87185 and Livermore, California 94550

for the United States Department of Energy

under Contract DE-AC04-94AL85000

Approved for public release; distribution is unlimited.

(n)
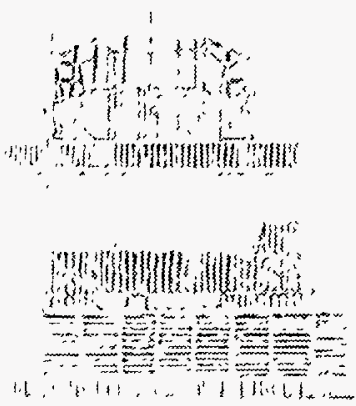

:

Uilu

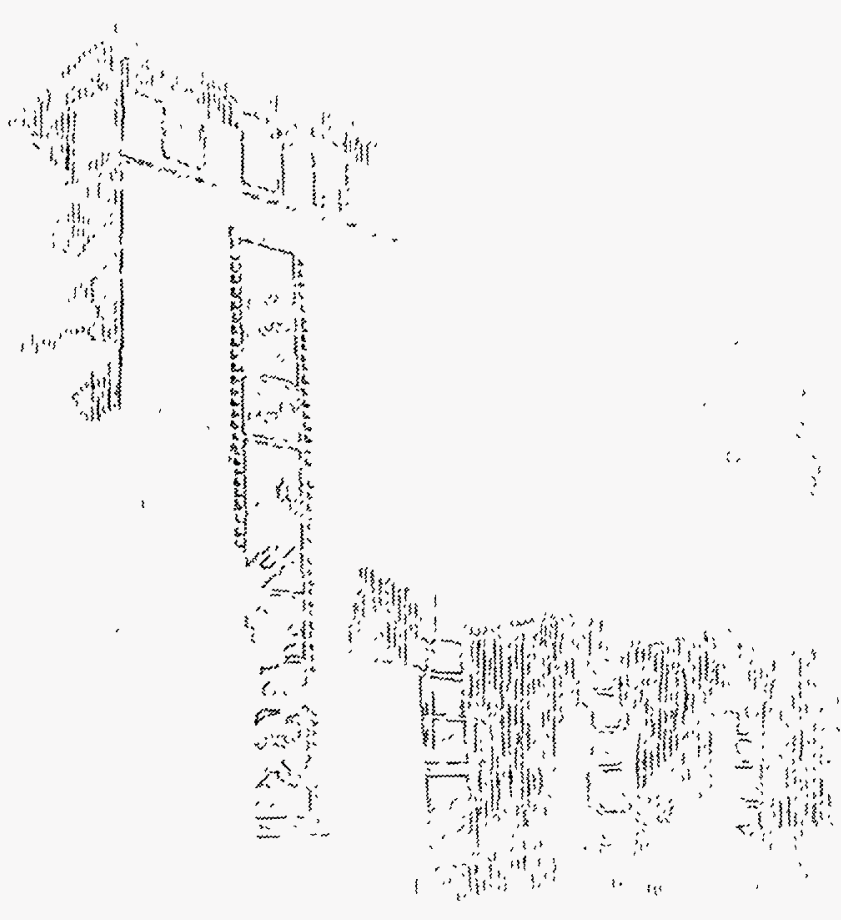


Issued by Sandia National Laboratories, operated for the United States Department of Energy by Sandia Corporation.

NOTICE: This report was prepared as an account of work sponsored by an agency of the United States Government. Neither the United States Government nor any agency thereof, nor any of their employees, nor any of their contractors, subcontractors, or their employees, makes any warranty, express or implied, or assumes any legal liability or responsibility for the accuracy, completeness, or usefulness of any information, apparatus, product, or process disclosed, or represents that its use would not infringe privately owned rights. Reference herein to any specific commercial product, process, or service by trade name, trademark, manufacturer, or otherwise, does not necessarily constitute or imply its endorsement, recommendation, or favoring by the United States Government, any agency thereof or any of their contractors or subcontractors. The views and opinions expressed herein do not necessarily state or reflect those of the United States Government, any agency thereof or any of their contractors.

Printed in the United States of America. This report has been reproduced directly from the best available copy.

Available to DOE and DOE contractors from

Office of Scientific and Technical Information

PO Box 62

Oak Ridge, TN 37831

Prices available from (615) 576-8401, FTS 626-8401

Available to the public from

National Technical Information Service

US Department of Commerce

5285 Port Royal Rd

Springfield, VA 22161

NTIS price codes

Printed copy: A03

Microfiche copy: A01

\section{DISCLAIMER}

This report was prepared as an account of work sponsored by an agency of the United States Government. Neither the United States Government nor any agency thereof, nor any of their employees, makes any warranty, express or implied, or assumes any legal liability or responsibility for the accuracy, completeness, or usefulness of any information, apparatus, product, or process disclosed, or represents that its use would not infringe privately owned rights. Reference herein to any specific commercial product, process, or service by trade name, trademark, manufacturer, or otherwise does not necessarily constitute or imply its endorsement, recommendation, or favoring by the United States Government or any agency thereof. The views and opinions of authors expressed herein do not necessarily state or reflect those of the United States Government or any agency thereof. 
SAND95-2790

Distribution

Unlimited Release

Printed December 1995

Category UC-706

\title{
Local Tetrahedron Modeling of Microelectronics Using the Finite- Volume Hybrid-Grid Technique
}

\author{
Douglas J. Riley and C. David Turner \\ Radiation and Electromagnetic Analysis Department \\ Sandia National Laboratories \\ Albuquerque, New Mexico 87185-1166
}

\begin{abstract}
The finite-volume hybrid-grid (FVHG) technique uses both structured and unstructured grid regions in obtaining a solution to the time-domain Maxwell's equations. The method is based on explicit time differencing and utilizes rectilinear finite-difference time-domain (FDTD) and nonorthogonal finite-volume time-domain (FVTD). The technique directly couples structured FDTD grids with unstructured FVTD grids without the need for spatial interpolation across grid interfaces. In this paper, the FVHG method is applied to simple planar microelectronic devices. Local tetrahedron grids are used to model portions of the device under study, with the remainder of the problem space being modeled with cubical hexahedral cells. The accuracy of propagating microstrip-guided waves from a low-density hexahedron region through a high-density tetrahedron grid is investigated.
\end{abstract}

\section{MASTER}




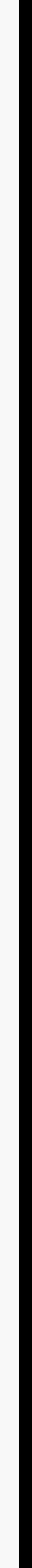




\section{Contents}

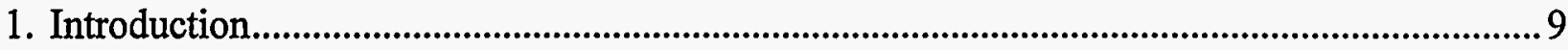

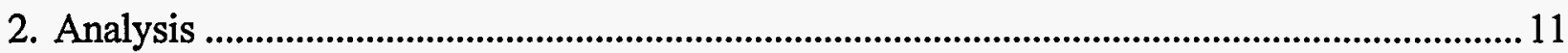

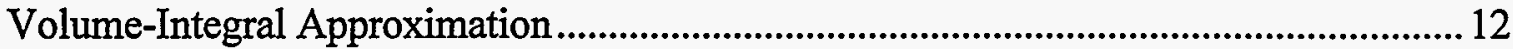

Area-Integral Approximation ...............................................................................................13

Time-Advancement Scheme ...................................................................................................... 13

FDTD Contribution and Edge-Projected Field ...................................................................16

Boundary-Condition Implementation for Open Conductors...............................................17

Grid Generation and Selection of Time Steps...................................................................... 18

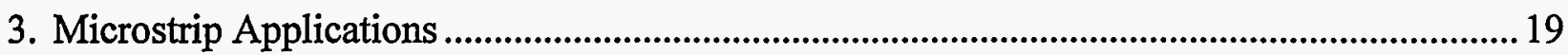

Uniform Microstripline ..................................................................................................... 19

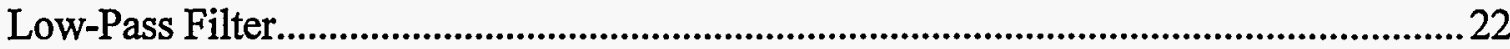

Microstrip Patch Antenna.................................................................................................24

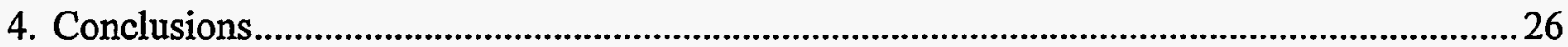

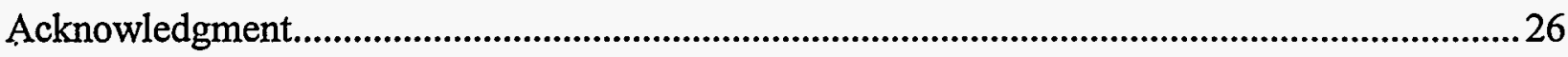

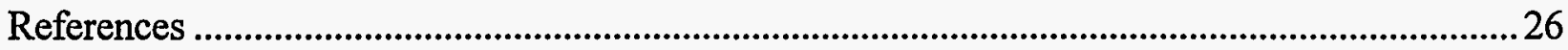




\section{Illustrations}

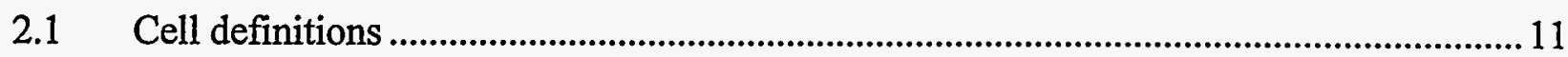

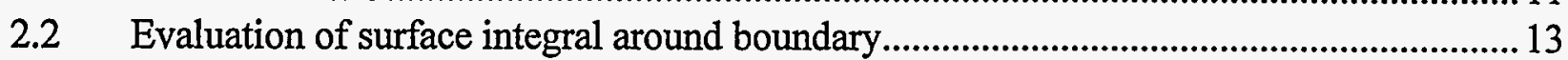

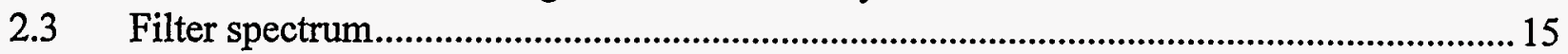

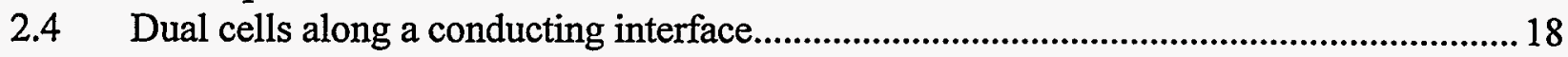

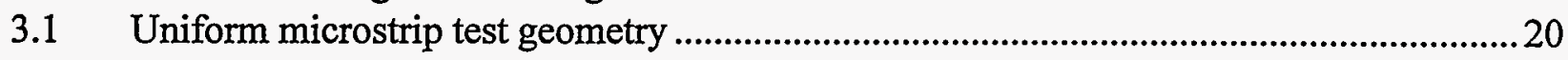

3.2 Portion of unstructured grid for Fig. 3.1 showing tetrahedra region, which extends from one hexahedral cell-width above the power trace to the ground plane......................20

3.3 Results at input and output planes based on all hexahedral (FDTD) and embedded tetrahedral (FVHG) cells .........................................................................................21

3.4a Difference of Fig. 3.3 results at input observer ........................................................... 21

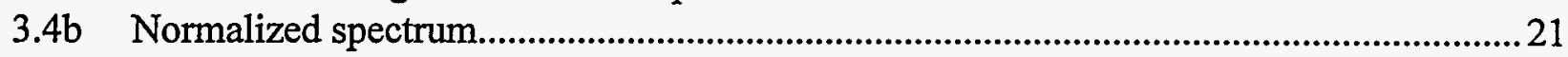

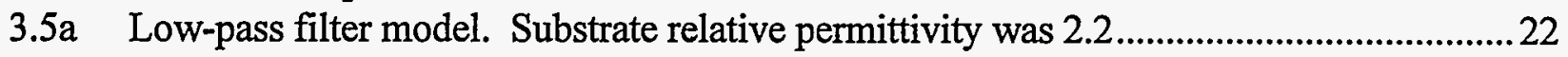

3.5b A section of the tetrahedra grid that encloses the low-pass filter......................................22

3.6 Full unstructured grid for low-pass filter including connecting traces at input and output ports. Tetrahedra region shown in Fig. $3.5 \mathrm{~b}$ is enclosed in the mid-section.........23

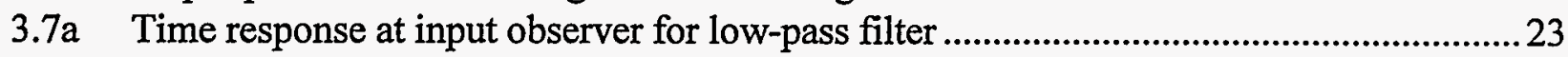

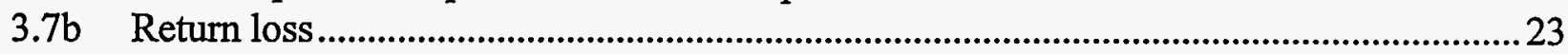

3.8a Time response at output observer for low-pass filter ....................................................... 23

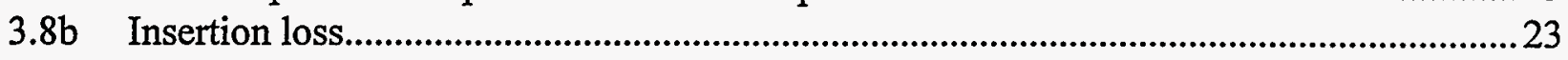

3.9 Model of patch antenna. Substrate permittivity was 2.2. The input port is at the trace "stub" on the left side of the figure.

3.10 Partial grid of patch antenna. The feeding trace, modeled with uniform quadrilateral shell elements, resides in the low-density hexahedron mesh and connects to the input port of the patch antenna. At this point, the grid transitions to a dense triangularelement surface mesh surrounded by tetrahedral elements ..............................................25

3.11a Time response at input observer for patch antenna ..............................................................2

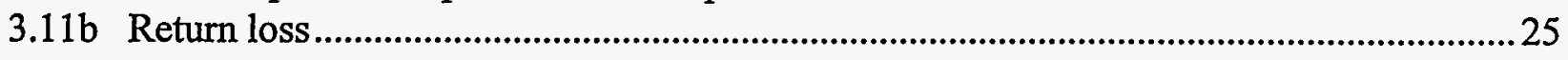

\section{Tables}

2.1 Effect of time average on uniform-grid Courant condition...........................................16 


\section{Local Tetrahedron Modeling of Microelectronics Using the Finite-Volume Hybrid-Grid Technique}

\section{Introduction}

Transient finite-difference, finite-element, and finite-volume methods represent powerful tools for the analysis of complex geometries. The finite-difference time-domain (FDTD) method introduced by Yee nearly 30 years ago [1] has been extensively applied to a wide variety of problems that range from microelectronics to biomedical applications. An extensive collection of references can be found in [2]. The standard FDTD method is mature, conceptually simple, and computationally efficient but possesses the well-known topological limitation of staircasing [3]. Finite-element and finite-volume methods provide increased geometrical flexibility by conforming elements to complex features, but often at a prohibitively high computational cost. Finiteelement methods (FEM) have traditionally been applied in the frequency domain to electrostatics, magnetostatics, and interior dynamic problems [4], but more general application is becoming possible through the use of edge elements and accurate absorbing boundary conditions [5]. Finite-element formulations based on explicit and implicit time-differencing methods are also under development [6,7]. Published work on transient finite-volume techniques has been based on the application of computational fluid-dynamics (CFD) methods to Maxwell's equations [8] and on a direct generalization of rectilinear FDTD to arbitrary convex polyhedrons [9-15]. Both techniques use explicit time differencing, but the CFD-based methods define the vector electric and magnetic fields on a single grid, whereas the generalized-Yee formulations use two interleaved grids referred to as primary and dual grids. It is noted that explicit finite-element formulations using Whitney elements applied to Maxwell's two curl equations also use a dual-type, secondary grid to evaluate the electric field along edges [6].

Much of the previous work involving time-domain volumetric solvers applied to open-region problems used mapped-meshing techniques. Primary grids of this type consist of skewed hexahedral and/or wedge elements. The grids are generally block structured but can, of course, be referenced in an unstructured manner. An example of mapped-meshing applied to microstrip problems consists of forming a surface grid on a planar geometry, and then extruding the surface, or shell, elements into solid elements. The resulting grid is considered to be 2-1/2 dimensional because the grid can not accommodate geometry variations along the extrusion direction (although different materials can be incorporated along this direction). The technique has been successfully applied by Gedney and Lansing [15]. For general geometries, mapped-meshing requires mapping, or projecting, one surface onto another. Geometries must be zoned using this approach, generality is limited, and user intervention is often high. Free-meshing, on the other hand, provides increased geometrical flexibility and generally lower user intervention. The resulting grids typically consist of tetrahedral elements, although fully free-meshed hexahedral grids are possible in some situations [16,17]. Tetrahedral grids are computationally expensive because cell count can become exceedingly high for practical problems. Compared to cubical 
hexahedral grids (FDTD), filling the same volume with free-meshed tetrahedra that possess an average edge length close to that of the hexahedral cells will lead to approximately eight times the number of cells. Consequently, it is desirable to minimize the number of tetrahedra in the problem space. The present paper addresses using local tetrahedron grids interfaced with FDTD grids and applied to microelectronics.

The software package VOLMAX (Volumetric Maxwell solver) is based on the finite-volume hybrid-grid (FVHG) technique. This technique permits non-orthogonal, unstructured grids to be embedded in traditional rectangular-cell, structured-grid, FDTD [13,14]. The FVHG method enables high complexity regions to be modeled and gridded using commercial solid-modeling software. The resulting complex unstructured grid is interfaced to simple FDTD cells without spatial interpolation across the grid interface. This is accomplished by forcing the unstructured grid to terminate on a closed rectangular surface [14]. The FVHG technique is particularly useful for open-region problems and/or geometries with local regions of high complexity. The method has been shown to provide high accuracy in scattering applications.

Microelectronics applications are well suited to an FVHG formulation. This is because uniform microstrips are often used at the input and output ports of the device under study. To obtain accurate $S$-parameter data, these microstrips usually extend a long distance and require accurate absorbing boundary conditions at the line terminations. This portion of the problem can be modeled accurately and efficiently using FDTD. However, the device under study may be of high complexity and require a conforming grid. This complex region can be designed using solid-modeling software and then gridded with free-meshed, tetrahedral elements. The resulting hybrid-grid will lead to an accurate and efficient description of the problem.

The finite-volume portion of the FVHG solver [13] is based on a generalization/simplification of the modified finite-volume technique introduced in [9]. A (variably dissipative) time-averaging scheme is used to obtain long-term stability. Achieving stability for tens-of-thousands of time steps on arbitrary grids with explicit (and even implicit), time-domain, finite volume and finite element schemes without using dissipation remains an open research issue across many disciplines. It is noted that the non-dissipative discrete surface integration (DSI) method [10] has been found to delay, but not eliminate, late-time growth on complex grids [13]. The technique used by the FVHG method permits either no dissipation, or a moderate amount of dissipation, depending on the grid complexity. Unfortunately, the "right amount" of dissipation is generally not known a priori, but guidelines are provided in the present paper that have been found to be successful for complex tetrahedron grids.

This paper provides an overview of the finite-volume algorithm, the time-integration scheme, boundary condition implementation, grid generation, and time-step definition. Three simple microstrip applications with well-known FDTD solutions are examined. The examples demonstrate the accuracy that can be obtained by launching microstrip-guided waves on cubical hexahedron (FDTD) grids and through a dense, unstructured linear tetrahedra region. More complex geometries that fully exploit the power of the FVHG approach to microelectronics will be topics of future papers. 


\section{Analysis}

The finite-volume method can accommodate arbitrary convex polyhedron-element primary grids. The vector magnetic fields are defined at the centroids of the primary cells, whereas the vector electric fields are defined at their vertices. Dual cells contain the vertices. The dual cells can be extremely complex and typically possess many faces, edges and nodes. A preprocessor builds the dual grid from the primary grid. The primary grid is equivalently labeled an $\mathbf{H}$-grid, whereas the dual grid is equivalently labeled an E-grid. A typical H-grid tetrahedral element, referenced as $\mathbf{H}_{i}^{\mathbf{t}}$, is shown in Fig. 2.1 (superscript $\mathbf{t}$ denotes total field). Dual cells (not shown) surround the four vertices and are referred to as $\mathbf{E}_{j}^{\mathbf{t}}, j=1-4$. The dual edge, $\mathbf{s}_{l}^{e}$, connects the centroids of primary-cell, $i$, with a neighboring cell, $k$. The primary edge, $\mathbf{s}_{p}^{h}$, connects $\mathbf{E}_{1}^{\mathbf{t}}$ and $\mathbf{E}_{2}^{\mathbf{t}}$. The volume of primary cell, $i$, is denoted $V_{i}^{h}$, while the volume of dual cell, $j$, is denoted $V_{j}^{e}$. The area of face, $l$, on primary cell, $i$, is denoted by $A_{i, l}^{h}$, while the area of face, $p$, on dual cell, $j$, is denoted $A_{j, p}^{e} \cdot \mathbf{n}_{i, l}^{h}$ and $\mathbf{n}_{j, p}^{e}$ denote outward normals for each face of the primary and dual cells, respectively.

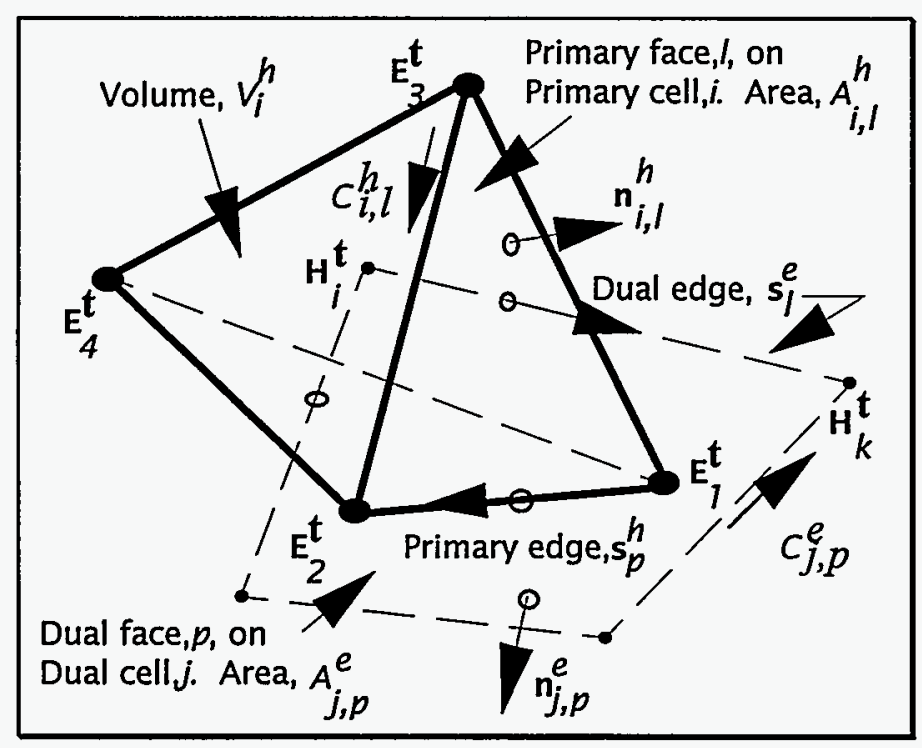

Maxwell's equations are given by

$$
\begin{aligned}
& \varepsilon \frac{\partial}{\partial t} \mathbf{E}^{\mathbf{t}}+\sigma_{e} \mathbf{E}^{\mathbf{t}}=\nabla \times \mathbf{H}^{\mathbf{t}} \\
& \mu \frac{\partial}{\partial t} \mathbf{H}^{\mathbf{t}}+\sigma_{m} \mathbf{H}^{\mathbf{t}}=-\nabla \times \mathbf{E}^{\mathbf{t}}
\end{aligned}
$$

where, $\varepsilon, \sigma_{e}, \mu$, and $\sigma_{m}$ denote electric permittivity, electric conductivity, magnetic permeability, and magnetic conductivity, respectively. By integrating over each cell on the primary and dual grids, the finite-volume time-domain (FVTD) form of Maxwell's equations is

Fig. 2.1. Cell definitions.

$$
\widetilde{\varepsilon}_{j} \frac{\partial}{\partial t} \iiint_{V_{j}^{e}} \mathbf{E}^{\mathbf{t}} d V_{j}^{e}+\widetilde{\sigma}_{e_{j}} \iiint_{V_{j}^{e}} \mathbf{E}^{\mathbf{t}} d V_{j}^{e}=\sum_{p} \iint_{A_{j, p}^{e}} \mathbf{n}_{j, p}^{e} \times \mathbf{H}^{\mathbf{t}} d A_{j, p}^{e}
$$

$$
\mu_{i} \frac{\partial}{\partial t} \iiint_{V_{i}^{h}} \mathbf{H}^{\mathbf{t}} d V_{i}^{h}+\sigma_{m_{i}} \iiint_{V_{i}^{h}} \mathbf{H}^{\mathbf{t}} d V_{i}^{h}=-\sum_{l} \iint_{A_{i, l}^{h}} \mathbf{n}_{i, l}^{h} \times \mathbf{E}^{\mathbf{t}} d A_{i, l}^{h}
$$


All materials are defined relative to primary cells. For magnetic materials this is the natural definition. However, electric materials are associated with the spatially staggered dual cells and therefore the permittivity and electric conductivity require averaging. The average values are denoted by $\widetilde{\varepsilon}_{j}$ and $\widetilde{\sigma}_{e_{j}}$, respectively. These are defined as follows. The average permittivity on dual face, $p$, is defined by

$$
\widetilde{\varepsilon}_{p}^{f}=\frac{\sum_{n} \varepsilon_{n} V_{n}^{h}}{\sum_{n} V_{n}^{h}}
$$

where the sums are taken over the primary cells associated with the dual face. The average permittivity for the dual $c e l l, j$, is constructed by forming

$$
\widetilde{\varepsilon}_{j}=\frac{\sum_{p} \widetilde{\varepsilon}_{p}^{f}}{N_{j}^{f}}
$$

where the sum is taken over all dual faces on dual cell, $j$, and $N_{j}^{f}$ denotes the number of faces on the cell. The electric conductivity is similarly defined.

\section{Volume-Integral Approximation}

The volume integrals are easily evaluated by assuming the vector field remains constant throughout the cell volume. This leads to the following equations

$$
\begin{aligned}
& \widetilde{\varepsilon}_{j} \frac{\partial}{\partial t} \mathbf{E}^{\mathbf{t}}+\widetilde{\sigma}_{e_{j}} \mathbf{E}^{\mathbf{t}}=\frac{1}{V_{j}^{e}} \sum_{p} \iint_{A_{j, p}^{e}} \mathbf{n}_{j, p}^{e} \times \mathbf{H}^{\mathbf{t}} d A_{j, p}^{e} \\
& \mu_{i} \frac{\partial}{\partial t} \mathbf{H}^{\mathbf{t}}+\sigma_{m_{i}} \mathbf{H}^{\mathbf{t}}=-\frac{1}{V_{i}^{h}} \sum_{l} \iint_{A_{i, l}^{h}} \mathbf{n}_{i, l}^{h} \times \mathbf{E}^{\mathbf{t}} d A_{i, l}^{h}
\end{aligned}
$$

A first-order approximation to the vector fields within cells can be constructed by expanding the fields in a first-order Taylor series and applying the relationship $\iiint \nabla \Psi d V=\iint \Psi \mathbf{n} d A$, where $\Psi$ denotes a scalar field and $\mathbf{n}$ denotes an outward normal. This relationship enables the field derivatives to be easily evaluated. The results in this paper are based on constant fields throughout the cell volume. The effect of higher-order representations will be examined in a future paper. 


\section{Area-Integral Approximation}

Similar to Madsen and Ziolkowski [9], the cross product in the area integral is expanded as a vector triple product at each vertex and an average value for the integral is constructed by summing over all vertices of the face, dividing by the number of vertices, and multiplying by the face area (which is not unique for non-planar faces). Note that for tetrahedral elements the faces are triangles, which leads to the following simplification (cf. Fig. 2.2):

$$
\begin{aligned}
& \iint_{i, l} \mathbf{n}_{i, l}^{h} \times \mathbf{E}^{\mathbf{t}} d A l \approx-\frac{1}{3} \sum_{p} \mathbf{E}_{p}^{\mathbf{t}} \times\left(\frac{\mathbf{s}_{p 1}^{h} \times \mathbf{s}_{p 2}^{h}}{\left|\mathbf{s}_{p 1}^{h} \times \mathbf{s}_{p 2}^{h}\right|}\right) \frac{1}{2}\left|\mathbf{s}_{p 1}^{h} \times \mathbf{s}_{p 2}^{h}\right|= \\
& \quad-\frac{1}{6} \sum_{p} \mathbf{E}_{p}^{\mathbf{t}} \times \mathbf{s}_{p 1}^{h} \times \mathbf{s}_{p 2}^{h} \approx \frac{1}{6} \sum_{p}\left(\left(\widetilde{\mathbf{E}}^{\mathbf{t}} \cdot \mathbf{s}_{p 1}^{h}\right) \mathbf{s}_{p 2}^{h}-\left(\widetilde{\mathbf{E}}^{\mathbf{t}} \cdot \mathbf{s}_{p 2}^{h}\right) \mathbf{s}_{p 1}^{h}\right)
\end{aligned}
$$

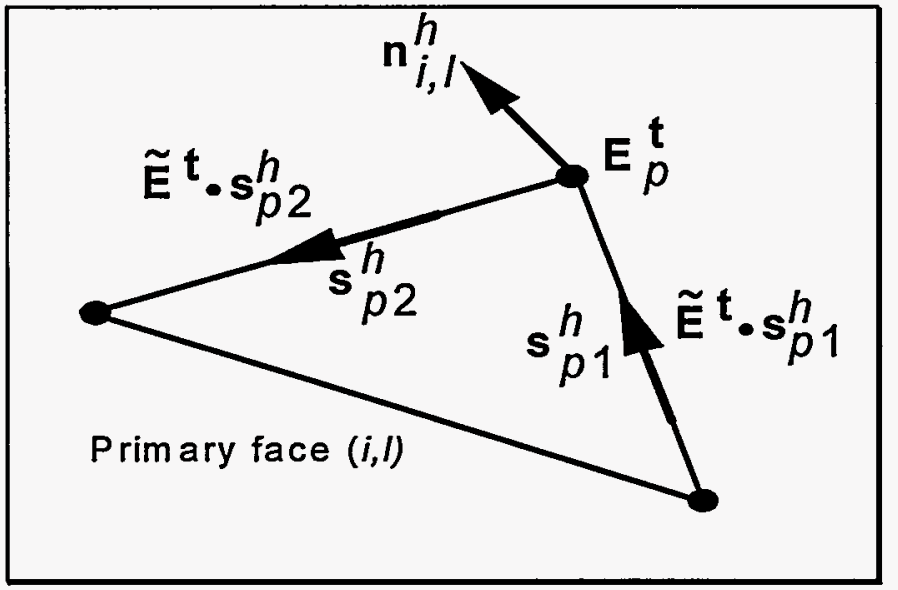

Fig. 2.2. Evaluation of surface integral around boundary.
The summation is taken over all vertices of primary face, $(i, l)$ (cf. Figs. 2.1, 2.2). The symbol,, represents an average field along the edge (defined below). An expression similar to (2.7) applies to quadrilateral faces, with the overall face normal often becoming an average direction. For faces with an arbitrary number of vertices, as is generally the case on the dual grid, the faces are subdivided into triangles from which the overall area is computed. For these faces, the face-normal generally becomes an average direction.

\section{Time-Advancement Scheme}

The application of explicit time-domain methods to complex cell shapes often leads to a well known, late-time, weak instability in the simulation $[13,18]$. This occurs in both finite-element and finite-volume formulations across many disciplines. The true nature of the instability is not fully understood but is believed to be due to a shifting of the eigenvalues of the discrete formulation relative to the continuous equations [18]. CFD-based methods rely on a dissipative term in the discrete equations to suppress problematic high-frequency modes [8]. The impact of this term as applied to Maxwell's equations has not been thoroughly addressed in the literature. For generalized-Yee formulations, the use of time-averaging to create a variably dissipative timeintegration scheme has been found to be effective [13]. However, dissipative time-integration can lead to "over-damping" of the simulation. This is particularly true when applied to lossless, 
closed, cavities. Problems that possess natural dissipation, such as radiation, can exhibit slightly enhanced attenuation in the higher frequencies due to the artificial dissipation, particularly as the grid resolution becomes less than approximately 20 cells per wavelength. For such problems, the artificial dissipation is often a second-order loss effect.

Traditional FDTD uses a second-order, centered time difference for the time derivatives. Second-order accuracy requires that the differences are offset in time between the electric and magnetic fields which gives rise to the well-known "leap-frog" time-differencing scheme. Applied to the magnetic field (with the electric field similar), the usual centered time difference is written as

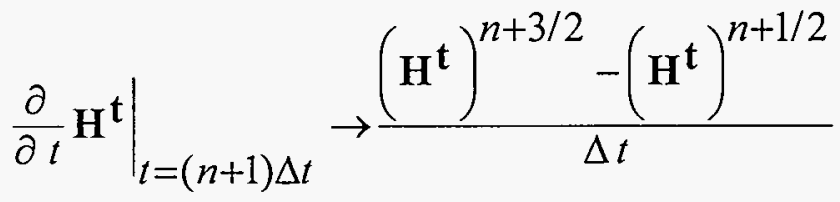

where $\Delta t$ denotes the time step. A (first-order) time average for $\left(\mathbf{H}^{\mathbf{t}}\right)^{n+1 / 2}$ is the following

$$
\left(\mathbf{H}^{\mathbf{t}}\right)^{n+1 / 2}=\frac{1}{\alpha+2}\left(\left(\mathbf{H}^{\mathbf{t}}\right)^{n+3 / 2}+\alpha\left(\mathbf{H}^{\mathbf{t}}\right)^{n+1 / 2}+\left(\mathbf{H}^{\mathbf{t}}\right)^{n-1 / 2}\right)
$$

where $\alpha \geq 0$, but practical values are dependent on $\Delta t$. The average corresponds to discrete time samples of the magnetic field. The magnitude of the Fourier Transform of the sampling function

$\frac{1}{\alpha+2}\left[\delta\left(t-\left(n+\frac{3}{2}\right) \Delta t\right)+\alpha \delta\left(t-\left(n+\frac{1}{2}\right) \Delta t\right)+\delta\left(t-\left(n-\frac{1}{2}\right) \Delta t\right)\right]$

where $\delta(\cdot)$ represents the Dirac delta function, is given by

$$
\left|\frac{1}{1+\frac{2}{\alpha}}+\frac{1}{1+\frac{\alpha}{2}} \cos (\omega \Delta t)\right|
$$

Here, $\omega$ corresponds to radian frequency. For values of $\alpha$ less than infinity, the time average provides increasing attenuation with increasing frequency (with practical upper frequencies selected considerably below the Nyquist sampling requirement). Consider a uniform grid in free space. Define $\Delta t=\Delta /(c N)$, where $c$ denotes the speed of light in vacuum, $\Delta$ denotes the spatial step throughout the grid, and $N$ represents a positive real constant required for stability. $\omega \Delta t$ can now be written as $(2 \pi / N)(\Delta / \lambda)$, where $\lambda$ denotes free-space wavelength. 
Writing the grid resolution as $\Delta=\lambda / M$, where $M$ is a pivsturce constant, leads to the result, $\omega \Delta t=2 \pi /(N \cdot M)$. A graph of Eq. 2.11 as a function of $N \cdot M$ is shown in Fig. 2.3.

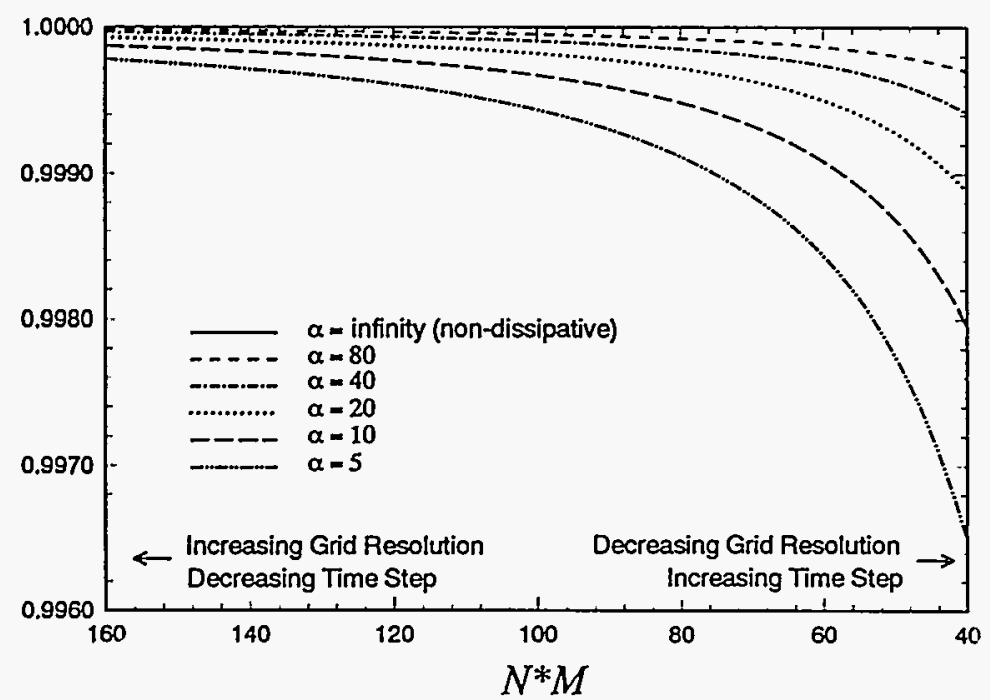

Fig. 2.3. Filter spectrum.

As an example of using Fig. 2.3, consider a grid resolution of 20 cells per wavelength $(M=20)$ and $N=2$ (which is typical for three-dimensional FDTD on a uniform grid); thus, $N \cdot M=$ 40. Choosing $\alpha=40$ leads to an attenuation value of 0.999400. At 80 cells per wavelength with the same $N$ and $\alpha$, the attenuation factor is 0.999963. Although these values may seem insignificant, they become cumulative in a timeadvancement scheme. Just as the standard Yee algorithm is dispersive, leading to increased phase error over propagation distance [19], the presented time-averaging scheme gives rise to frequency-dependent attenuation. Consider pulse propagation in one dimension over a distance of 100 cells with $N=2$; thus, 200 time steps are required to travel the line. Using $\alpha=40$, the frequency content of the signal at the end of the line is attenuated by approximately $12 \%(1 \mathrm{~dB})$ at 20 cells per wavelength and $0.8 \%(0.06 \mathrm{~dB})$ at 80 cells per wavelength compared to lossless propagation. These results were obtained by raising the appropriate value from Fig. 2.3 to the required number of time steps.

For fixed values of $\alpha$ and $M$, increasing $N$ (corresponding to decreasing $\Delta t$ ) leads to decreasing dissipation. Although this may appear desirable, the resulting dissipation may be insufficient to damp the growth of problematic high-frequency modes, and consequently, a smaller value of $\alpha$ may be required. For embedded, free-meshed, tetrahedron grids, numerical experiments have lead to the required relationship, $N \cdot \alpha \leq 80$, for long-term stability over tens-of-thousands of time steps. Taking $N \cdot \alpha$ in the range $60-80$ has generally been found to be successful.

The advancement equations based on time-averaging are the following:

$$
\begin{aligned}
& \left(\mathbf{E}_{j}^{\mathbf{t}}\right)^{n+1}\left[A_{e}-\frac{B_{e}}{\alpha+2}\right]= \\
& \frac{B_{e}}{\alpha+2}\left[\alpha\left(\mathbf{E}_{j}^{\mathbf{t}}\right)^{n}+\left(\mathbf{E}_{j}^{\mathbf{t}}\right)^{n-1}\right]+\frac{1}{V_{j}^{e}} \sum_{p} \iint_{A_{j, p}^{e}} \mathbf{n}_{j, p}^{e} \times \mathbf{H}^{\mathbf{t}} d A_{j, p}^{e}-\widetilde{\sigma}_{e_{j}} \mathbf{E}^{\mathbf{t}}
\end{aligned}
$$




$$
\begin{aligned}
& \left(\mathbf{H}_{i}^{\mathbf{t}}\right)^{n+3 / 2}\left[A_{h}-\frac{B_{h}}{\alpha+2}\right]= \\
& \frac{B h}{\alpha+2}\left[\alpha\left(\mathbf{H}_{i}^{\mathbf{t}}\right)^{n+1 / 2}+\left(\mathbf{H}_{i}^{\mathbf{t}}\right)^{n-1 / 2}\right]-\frac{1}{V_{i}^{h}} \sum_{l} \iint_{A_{i, l}^{h}} \mathbf{n}_{i, l}^{h} \times \mathbf{E}^{\mathbf{t}} d A_{i, l}^{h}-\sigma_{m_{i}} \mathbf{H}^{\mathbf{t}}
\end{aligned}
$$

where

$$
A_{e}=\left(\frac{\widetilde{\varepsilon}_{j}}{\Delta t}+\frac{\widetilde{\sigma}_{e_{j}}}{2}\right), \quad B_{e}=\left(\frac{\widetilde{\varepsilon}_{j}}{\Delta t}-\frac{\widetilde{\sigma}_{e_{j}}}{2}\right), \quad A_{h}=\left(\frac{\mu_{i}}{\Delta t}+\frac{\sigma_{m_{i}}}{2}\right), \quad B_{h}=\left(\frac{\mu_{i}}{\Delta t}-\frac{\sigma_{m_{i}}}{2}\right)
$$

The effect of the time average on the usual Courant condition for a uniform, free-space, threedimensional FDTD grid is easily established by performing a Fourier (von Neumann) stability analysis [20] of the advancement equations. The analysis is straightforward to implement and only the final result is presented here. In terms of the filter parameter, $\alpha$, the resulting equation for determining the relationship between the time and spatial steps is given by

$$
\beta^{4}(\alpha+1)^{2}+2 \beta^{3}\left[2(\alpha+2)^{2} \eta-\alpha(\alpha+1)\right]+\beta^{2}\left[\alpha^{2}-2(\alpha+1)\right]+2 \alpha \beta+1=0
$$

where $|\beta| \leq 1$ for stability, and $c \Delta t=\sqrt{\frac{\eta}{3}} \Delta$. Sample calculations are shown in Table 2.1.

\begin{tabular}{|cc|}
\hline$\alpha$ & $\sqrt{\eta}$ \\
\hline$\infty$ & 1 \\
\hline $\mathbf{8 0}$ & 0.975 \\
\hline $\mathbf{4 0}$ & 0.952 \\
\hline $\mathbf{1 0}$ & 0.833 \\
\hline $\mathbf{5}$ & 0.714 \\
\hline
\end{tabular}

Table 2.1

Effect of Time Average on UniformGrid Courant Condition
It is seen that the Courant condition slightly tightens with decreasing $\alpha$. Although it is not possible to derive a similar rigorous relationship for arbitrary unstructured grids, a similar conclusion has been observed numerically.

\section{FDTD Contribution and Edge-Projected Field}

Knowledge of the vector fields at cell vertices enables the calculation of an average field projected along connecting edges. Arithmetic or volumetric averaging can be used. However, the approach does not guarantee that the fields are divergence free on a single cell level, and the algorithm does not reduce identically to an FDTD form when cells become orthogonal. These issues are rectified by incorporating an FDTD correction to the edge-projected vector field. With reference to Fig. 2.1, Ampere's and Faraday's laws specialized to the field components normal to primary-face $(i, l)$, and dual-face, $(j, p)$, are 
$\widetilde{\varepsilon}_{p}^{f} \frac{\partial}{\partial t} \underset{A_{j, p}^{e}}{\iint_{p}} E_{p} d A_{j, p}^{e}+\widetilde{\sigma}_{e_{p}}^{f} \underset{A_{j, p}^{e}}{\iint_{j, p}} E_{p} d A_{j, p}^{e}=\underset{C_{j, p}^{e}}{\oint \widetilde{\mathbf{H}}^{\mathbf{t}}} \cdot \mathbf{d l} \mathbf{l}$

$\tilde{\mu}_{l}^{f} \frac{\partial}{\partial t} \iint_{A_{i, l}^{h}} H_{l} d A_{i, l}^{h}+\widetilde{\sigma}_{m_{l}}^{f} \iint_{A_{i, l}^{h}} H_{l} d A_{i, l}^{h}=-\underset{C_{i, l}^{h}}{\oint \widetilde{\mathbf{E}}^{\mathbf{t}}} \cdot \mathbf{d} \mathbf{l}$

Where $H_{l}$ and $E_{p}$ denote the (face) normal components of the magnetic and electric fields (traditional FDTD). The fields are assumed to remain constant over the area integrals. The timeaveraging scheme discussed in the previous section is used for the time derivatives. The "face average" permittivity and electric conductivity discussed above have been used (cf. Eq. (2.3)). In addition, a face average for the permeability $\left(\tilde{\mu}_{l}^{f}\right)$ and magnetic conductivity $\left(\widetilde{\sigma}_{m l}^{f}\right)$ has been introduced. This is because the evaluation face is located at the interface of two cells. The average permeability is defined by $\tilde{\mu}_{l}^{f}=\left(V_{i}^{h} \mu_{i}+V_{k}^{h} \mu_{k}\right) /\left(V_{i}^{h}+V_{k}^{h}\right)$, with $\widetilde{\sigma}_{m l}^{f}$ similar.

The magnetic field projected along dual-edge, $\mathbf{s}_{l}^{e}$, is finally constructed by forming

$$
\widetilde{\mathbf{H}}^{\mathbf{t}} \cdot \mathbf{s}_{l}^{e}=\frac{1}{2}\left(\mathbf{H}_{i}^{\mathbf{t}}+\mathbf{H}_{k}^{\mathbf{t}}\right) \cdot \mathbf{s}_{l}^{e}+\left[H_{l}-\frac{1}{2}\left(\mathbf{H}_{i}^{\mathbf{t}}+\mathbf{H}_{k}^{\mathbf{t}}\right) \cdot \mathbf{n}_{i, l}^{h}\right]\left(\mathbf{n}_{i, l}^{h} \cdot \mathbf{s}_{l}^{e}\right)
$$

This expression directly incorporates the FDTD term into the average vector field. Note that when $\left|\mathbf{n}_{i, l}^{h} \cdot \mathbf{s}_{l}^{e}\right|=1$, the expression returns only the FDTD result. Arithmetic averaging of the vector fields at the edge endpoints has been used. The average electric field, $\widetilde{\mathbf{E}} \mathbf{t}$, projected along primary-edge, $\mathbf{s}_{p}^{h}$, is similar. Note the use of edge-projected fields in Eqs. $(2.7,2.15,2.16)$.

\section{Boundary-Condition Implementation for Open Conductors}

When a primary grid is generated for an inhomogeneous region, nodes lie along the material interfaces (cf. Fig. 2.4). Dual cells associated with these nodes extend across the interface which is why material averaging is used. In the case of a conducting sheet, the boundary dual cells become split, either completely or partially, depending on the location of the primary-cell node. Direct computation for the vector electric field is no longer possible in the usual sense for these cells. Of course, the tangential component of the vector field is zero, but the normal component generally is not. A similar situation occurs with traditional FDTD, but due to its rectilinear nature, no complication occurs. However, a complication does occur with non-orthogonal cells whenever the computation of $\widetilde{\mathbf{E}}^{\mathbf{t}} \cdot \mathbf{s}_{p}^{h}$, where $\mathbf{s}_{p}^{h}$ denotes a primary edge with one node on the 
conductor, doesn't simplify to the FDTD term, $E_{p}$. In this case, the following alternative expression for $\widetilde{\mathbf{E}}^{\mathbf{t}} \cdot \mathbf{s}_{p}^{h}$ is used (noting $\widetilde{\mathbf{n}}_{s p} \times \mathbf{E}_{j}^{\mathbf{t}}=0$ )

$$
\begin{aligned}
& \widetilde{\mathbf{E}}^{\mathbf{t}} \cdot \mathbf{s}_{p}^{h}=\frac{1}{2}\left(\mathbf{E}_{i}^{\mathbf{t}}+\left(\widetilde{\mathbf{n}}_{s p} \cdot \mathbf{E}_{i}^{\mathbf{t}}\right) \widetilde{\mathbf{n}}_{s p}\right) \cdot \mathbf{s}_{p}^{h}+ \\
& {\left[E_{p}-\frac{1}{2}\left(\mathbf{E}_{i}^{\mathbf{t}}+\left(\widetilde{\mathbf{n}}_{s p} \cdot \mathbf{E}_{i}^{\mathbf{t}}\right) \widetilde{\mathbf{n}}_{s p}\right) \cdot \mathbf{n}_{j, p}^{e}\right]\left(\mathbf{n}_{j, p}^{e} \cdot \mathbf{s}_{p}^{h}\right) }
\end{aligned}
$$

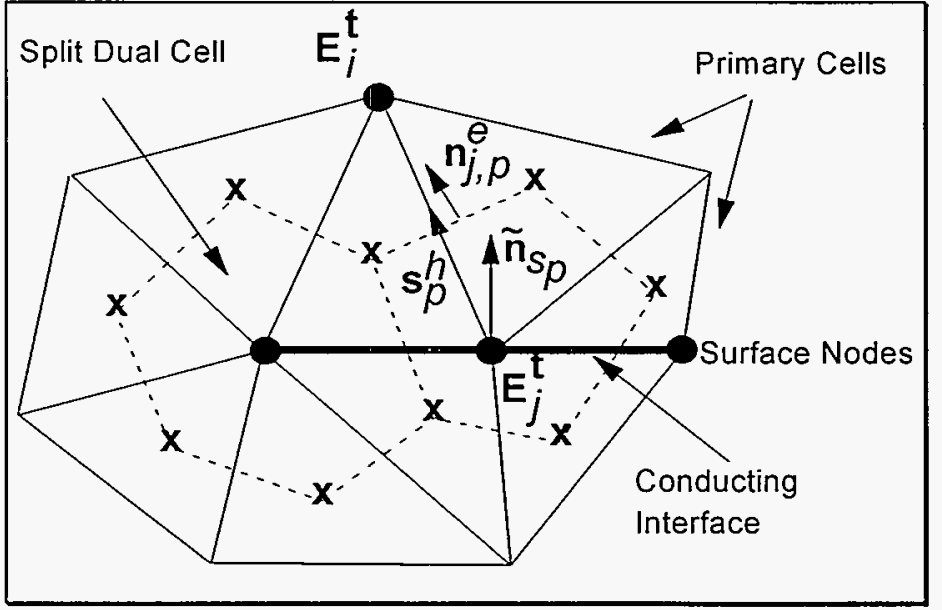

Fig. 2.4. Dual cells along a conducting interface.

where $\widetilde{\mathbf{n}}_{s p}$ denotes an average surface normal at the boundary node and $\mathbf{E}_{i}^{\mathbf{t}}$ denotes the known vector field associated with edge, $\mathbf{s}_{p}^{h}$. The average surface normal is constructed by forming a weighted average of neighboring face normals using only those faces residing on the conductor. Note that expression (2.18) incorporates the known zero tangential field at the boundary node, but relies upon the vector field off the surface to provide the estimate for the (surface) normal component. As be-
fore, the FDTD contribution is used to correct the (face) normal component (which has been found to be a critical contribution). Improved representations remain an active area of research.

\section{Grid Generation and Selection of Time Steps}

The FVHG algorithm implemented here uses the CAD package I-DEAS [21] to generate solid models, free-meshed linear tetrahedron grids, and/or mapped-mesh hexahedral/wedge grids. The method to terminate the tetrahedron grid and connect a structured FDTD grid can be found in Ref. [14]. The method is based on embedding the geometry of interest in a series of rectangular boxes that are partitioned with quadrilateral sub-surfaces. These surface partitions force the tetrahedron grid to terminate such that the surface nodes will spatially align with the nodes of the structured FDTD grid that will be connected. The creation of the proper surface partitions is easily automated within I-DEAS. The direct interface to hexahedral cells is realized through the use of seven-faced, six-sided elements called "wrapper cells." These cells are generated through the pre-processor as a consequence of the topology of a hexahedral element being interfaced to the bases of two tetrahedra. The wrapper completely encloses the unstructured region. The resulting grid (including the wrapper) represents the unstructured primary grid. The corresponding dual grid is constructed during the pre-processing phase. It is noted that because the use of wrapper cells enables the connection of mapped-mesh hexahedral elements to free-meshed tetrahedra, these two gridding methods can be logically combined within I-DEAS. 
The primary and dual grids are scanned to determine their maximum, minimum, and average edge lengths. The time step to be used in the unstructured region is related to the minimum edge length, but it can generally be chosen considerable larger than what the absolute smallest edge length would dictate. The following provides guidelines for the choice of the unstructured-grid time step obtained through numerical experiments.

Define the ratio of the maximum to minimum edge lengths on the primary and dual grids to be $R_{p}$ and $R_{d}$, respectively, and let $N_{\mathcal{U}}=\left[\left[\xi \max \left(\left[\left[R_{p}\right]\right],\left[\left[R_{d}\right]\right]\right)\right]\right]$, with [[·]] denoting

the closest integer greater than the argument, and $1 / 2 \leq \xi \leq 1$. Define $\Delta t_{u}=\Delta t_{S} / N_{\mathcal{U}}$, where $\Delta t_{u}$ denotes the time step in the unstructured grid and $\Delta t_{S}$ denotes the time step in the structured (FDTD) grid. The unstructured-grid time step becomes an integral sub-division of the FDTD time step which enables this region to be advanced several sub-time steps for each time step in the FDTD grid. Second-order time-interpolation has been found to be an effective means to time-couple the two regions [13]. Assuming a uniform, free-space FDTD grid with spatial step, $\Delta, \Delta t_{S}=\Delta /\left(c N_{S}\right)$. For this type of grid, $N_{S}$ is typically defined to be equal to 2 (but could be taken as small as $\sqrt{3})$. In a free-space unstructured region, $\Delta t_{\mathcal{u}}=\Delta /\left(c N_{S} N_{\mathcal{U}}\right)$. It is assumed that time averaging is only used in the unstructured region, and as previously shown, time averaging slightly tightens the Courant condition. This is why the parameter $\xi$ can not be uniquely defined. As the filtering effect increases (corresponding to decreasing $\alpha$ ), $\xi$ generally needs to be chosen toward the upper end of the defined range. Having defined the time step, the dissipation effect is easily estimated from Fig. 2.3 by setting $N=N_{U} \cdot N_{S}$.

\section{Microstrip Applications}

Three well known rectangular microstrip geometries have been selected to study the effect of local embedded tetrahedron grids on accuracy. Issues involving grid reflection at the hexahedrontetrahedron interfaces, propagation through dense tetrahedron regions, sub-time stepping, and the effect of dissipation in the unstructured grid, are examined through these examples.

\section{Uniform Microstripline}

As a first example, the simple microstrip shown in Fig. 3.1 was investigated. The substrate relative permittivity was 2.2. The geometry was modeled by a uniform collection of hexahedral elements with an edge length of $0.1985 \mathrm{~mm}$, along with an embedded tetrahedron region with an average edge length of $0.1985 \mathrm{~mm}$. The location and form of the tetrahedron region are shown in Figs. 3.1 and 3.2. The microstrip was locally terminated with a simple first-order Mur absorbing boundary condition $(A B C)$. Both the hexahedron and tetrahedron grids shown in Fig. 3.2 were created within I-DEAS and jointly define the unstructured grid (excluding the wrapper). Upon adding the wrapper during the preprocessing phase, the unstructured grid was embedded in a uniform FDTD mesh that measured $202 \times 68 \times 88$ cells in the $\mathrm{x}, \mathrm{y}$, and $\mathrm{z}$ directions, respectively. The FDTD cell size was also $0.1985 \mathrm{~mm}$. The FDTD mesh was terminated with second-order Mur boundaries. In the FDTD mesh, $N_{S}$ was set to 2, and $\alpha=\infty$. In the unstructured region, $N_{\mathcal{U}}=3$ (3 sub-time steps), and $\alpha=12$ (based on the previous guideline, $N_{S} \cdot N_{\mathcal{U}} \cdot \alpha \leq 80$ ). 


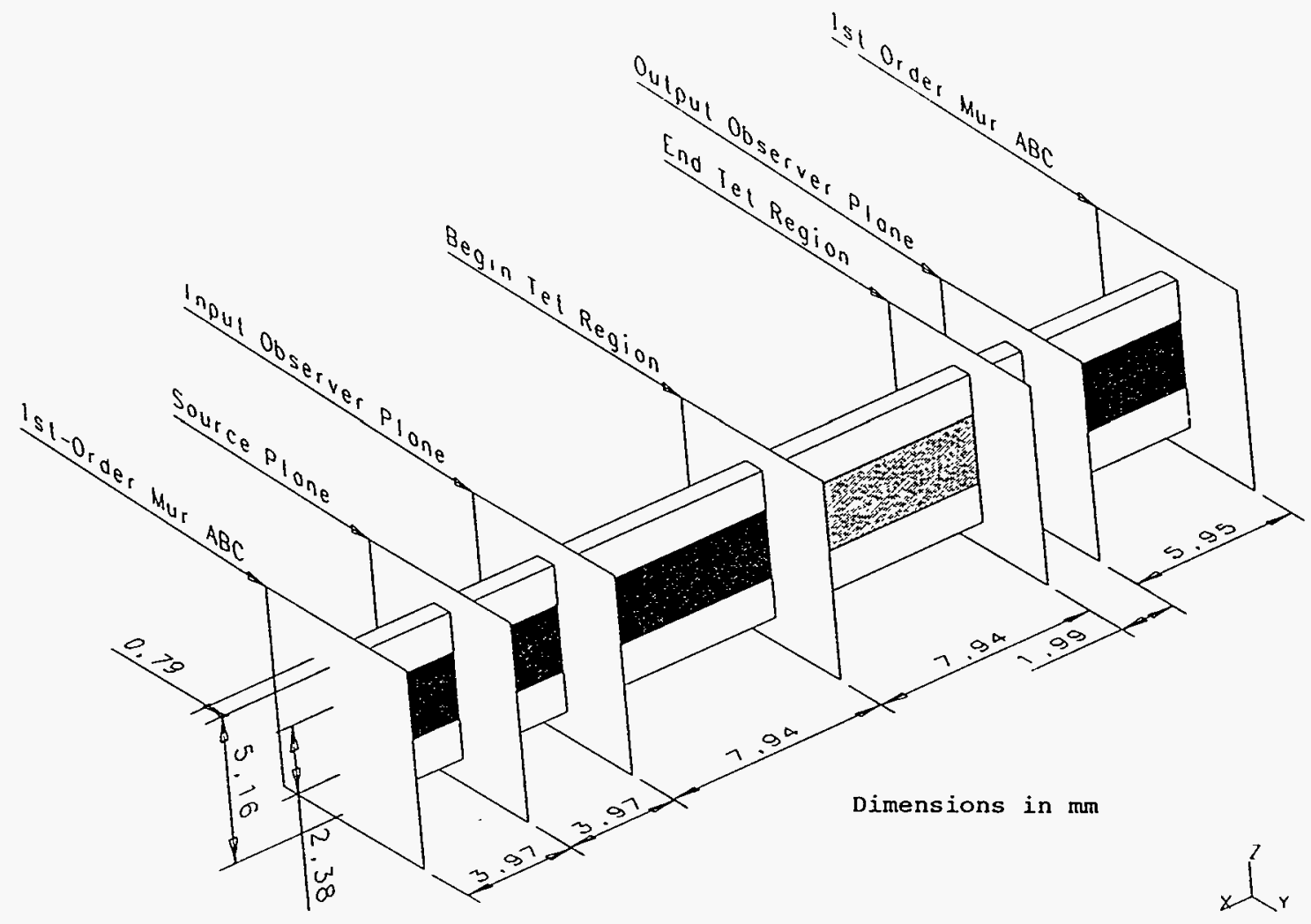

Fig. 3.1. Uniform microstrip test geometry. (Dimensions in $\mathrm{mm}$.)

The microstrip was driven by adding a gaussian source between the power and ground plitiss at the location shown in Fig. 3.1. Fig. 3.3 shows the voltage under the midpoint of the power trace at the input and output observer planes. A comparison is made by replacing the FVHG tetrahedron region with cubical hexahedral cells so that the finite-volume algorithm resorts exclusively to the FDTD term. Although this comparison mesh is now exclusively FDTD, the microstrip remains unstructured with the same values for $N_{\mathcal{U}}$ and $\alpha$; this permits a careful examination of guided-wave propagation through tetrahedrons and across hex-tet interfaces. As shown in Fig.

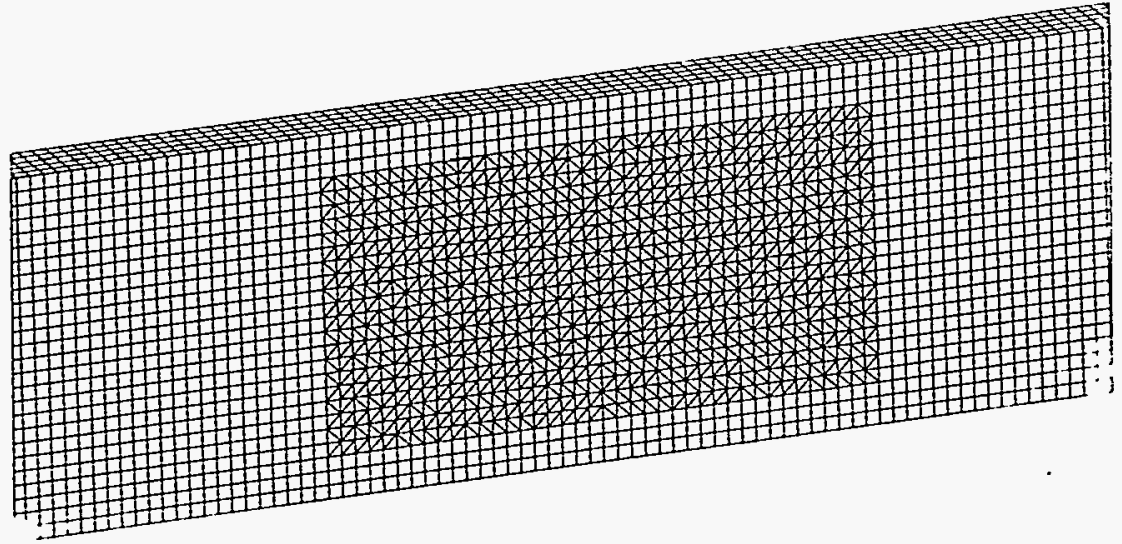

Fig. 3.2. Portion of unstructured grid for Fig. 3.1 showing tetrahedra region, which extends from one hexahedral cell-width above the power trace to the ground plane.
3.3, the results at the two observers are nearly identical for the two grid types. The only noticeable difference is a slight delay at the output observer when the tetrahedrons are present (FVHG results). This is most likely due to the increased cell density associated with the tetrahedrons. 


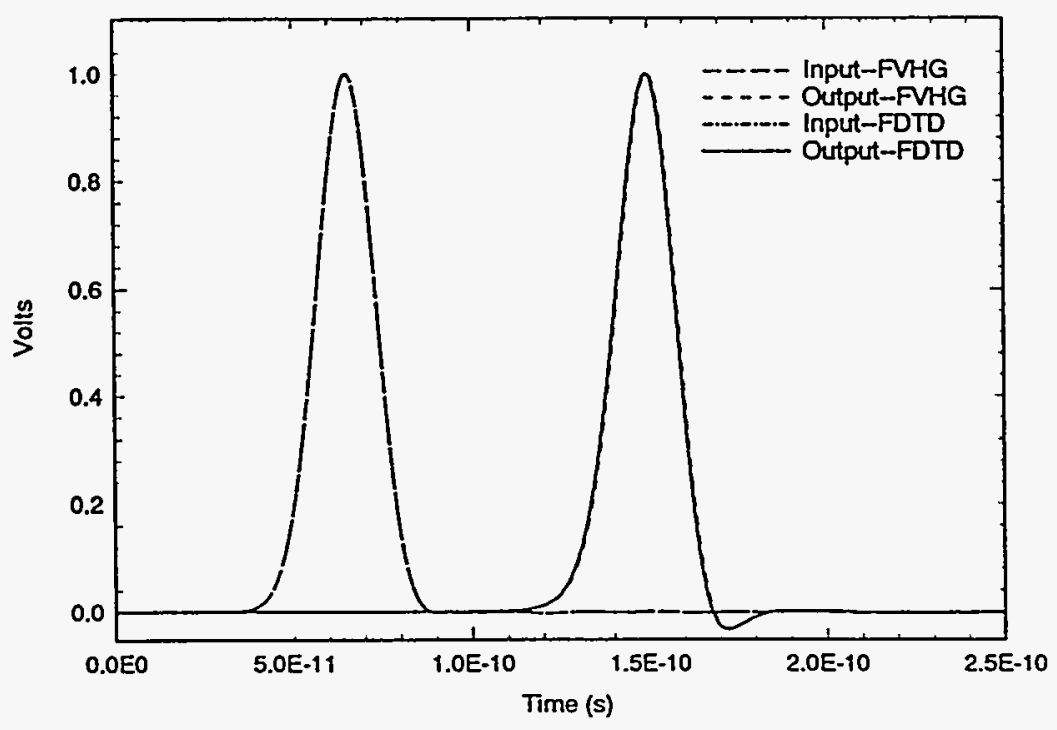

Fig. 3.3. Results at input and output planes based on all hexahedral (FDTD) and embedded tetrahedral (FVHG) cells.

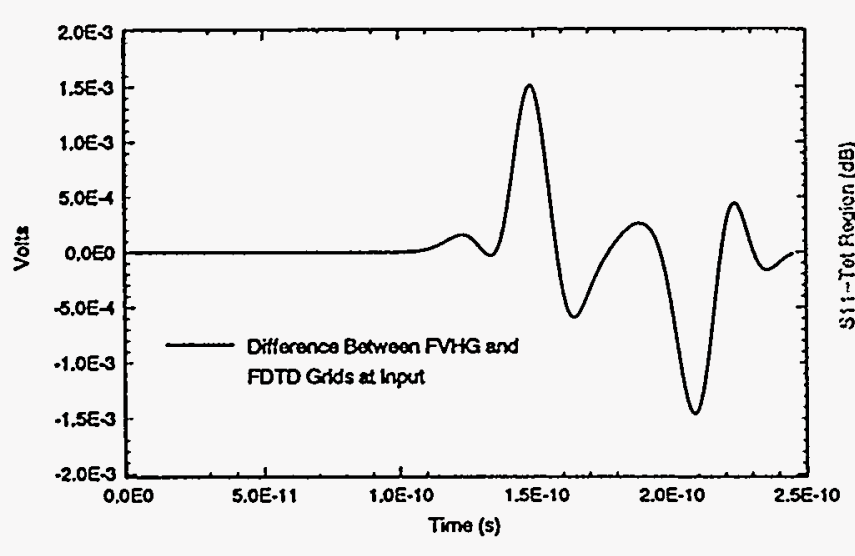

Fig. 3.4a. Difference of Fig. 3.3 results at input observer.

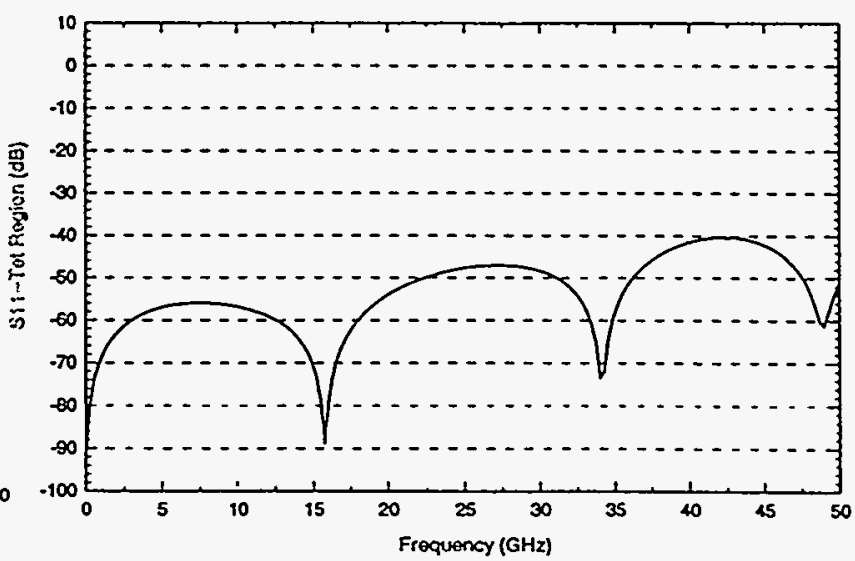

Fig. 3.4b. Normalized spectrum.

The difference between the FDTD and FVHG results at the input observer is shown in Fig. 3.4a. This reveals the subtle propagation differences between the grid types. The (peak) voltage difference is seen to be nearly three orders of magnitude below the peak voltage in Fig. 3.3. It is believed that this can be further reduced with improved boundary conditions for open conductors (cf. Section 2). The spectrum of the difference field, normalized to the incident-field spectrum, is shown in Fig. 3.4b. This result can be considered the return loss $(S 11)$ of the tetrahedra region. In the substrate, the FVHG grid resolution is approximately 20 "cells" per wavelength at $50 \mathrm{GHz}$ based on the maximum of the average edge lengths of the primary and dual grids. 


\section{Low-Pass Filter}

The second example is the well known low-pass filter originally studied by Sheen, et al., [22]. A portion of the filter modeled in the current paper is shown in Fig. 3.5a. Linear microstrip lines connect to the input and output ports. The geometry shown in Fig. 3.5a was modeled with tetrahedral elements with an average edge length of $0.264667 \mathrm{~mm}$. This portion of the overall grid contained approximately 50,000 tetrahedra and is shown in Fig. 3.5b. Uniform hexahedral elements with an edge length of $0.264667 \mathrm{~mm}$ were used to model the microstrips that connect to the input and output ports depicted in Fig. 3.5a; these element were created using mapped-mesh techniques within I-DEAS. It is noted that some modeling flexibility has been lost by forcing the tetrahedra to interface with these hexahedral elements; this accounts for the slight shifting of the port locations and the reduced trace widths relative to the actual geometry shown by Sheen, et al., [22]. The complete unstructured grid, including the wrapper, is shown in Fig. 3.6. This grid was embedded in a large, uniform FDTD grid that measured $180 \times 66 \times 148$ cells. Second-order Mur boundaries terminated the FDTD grid. The FDTD cell size was $0.264667 \mathrm{~mm}$. The microstrip was driven and terminated similar to the previous example. The source plane was located 16 cells from the left side of the unstructured grid shown in Fig. 3.6. The number of subtime steps in the unstructured grid was 4 , and the stabilization parameter was set to 10 .

The location of the observer on the input microstrip was under the midpoint of the power trace and placed three cells from the edge of the left side of the unstructured grid shown in Fig. 3.6. The observer on the output microstrip was located 16 cells from the right side of this grid. The operating range for this circuit is less than $10 \mathrm{GHz}$. The time response at the input observer is shown in Fig. 3.7a. The corresponding result for $S 11$ is shown in Fig. 3.7b. Results at the output observer are shown in Figs. 3.8a,b. The simulation ran for 20,000 time steps in the u: :actured grid (5,000 in 'he !'DTD grid) and required approximately 8 hours using 8 processors al a Sun Sparcserver 1() (with $50 \mathrm{MHz}$ Sparc-51 chips, which are no: ... (100 processors).

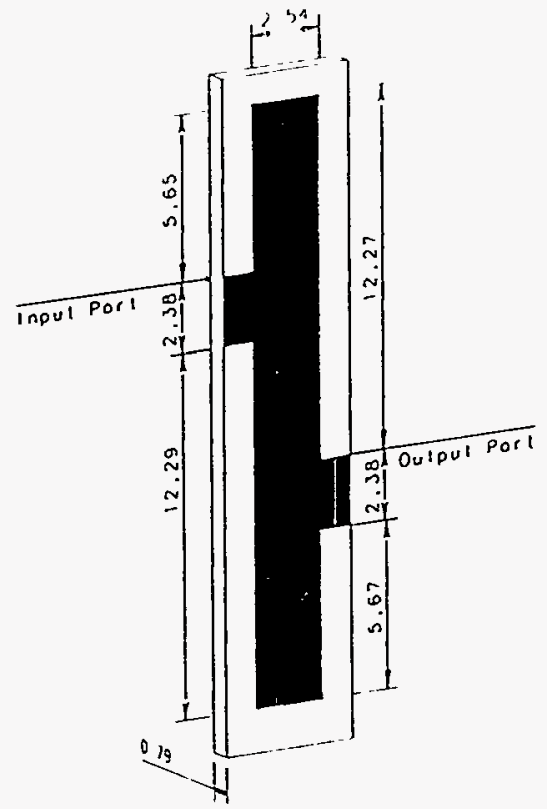

Fig. j.Sa. Low-pass filter model. Substrate relative permittivity was 2.2 . (Dimensions in $\mathrm{mm}$.)

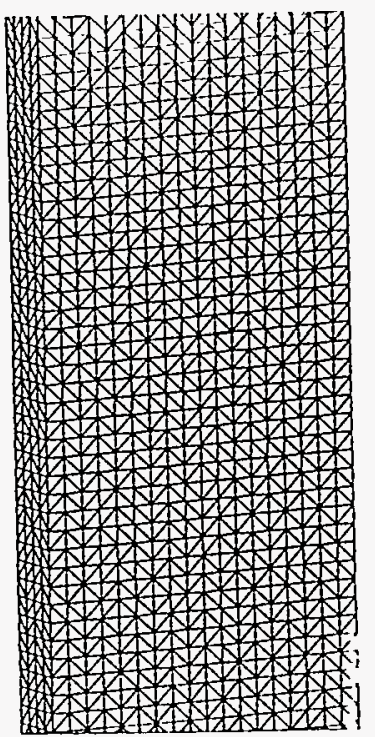

Fig. 3.5b. A section of the tetraheris in: : that encloses the low-p ... in: : 


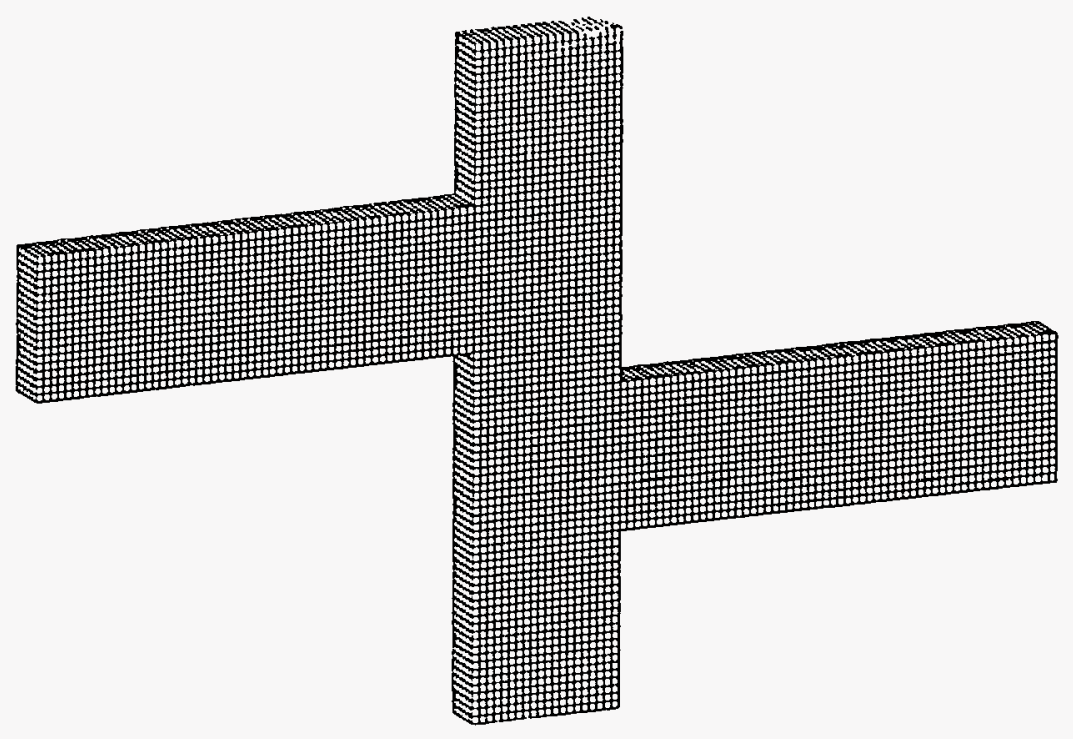

Fig. 3.6. Full unstructured grid for low-pass filter including connecting traces at input and output ports. Tetrahedra region shown in Fig. 3.5b is enclosed in the mid-section.

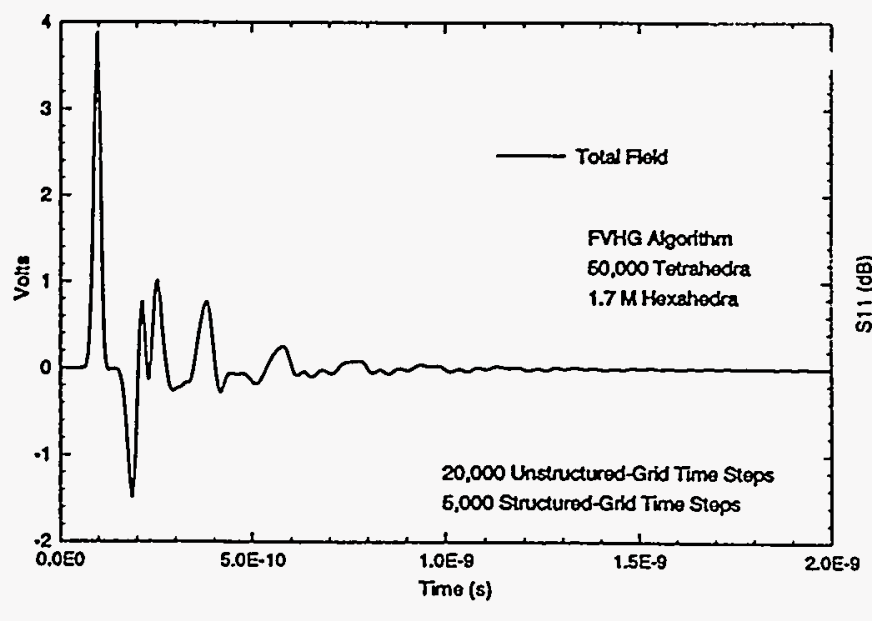

Fig. 3.7a. Time response at input observer for low-pass filter.

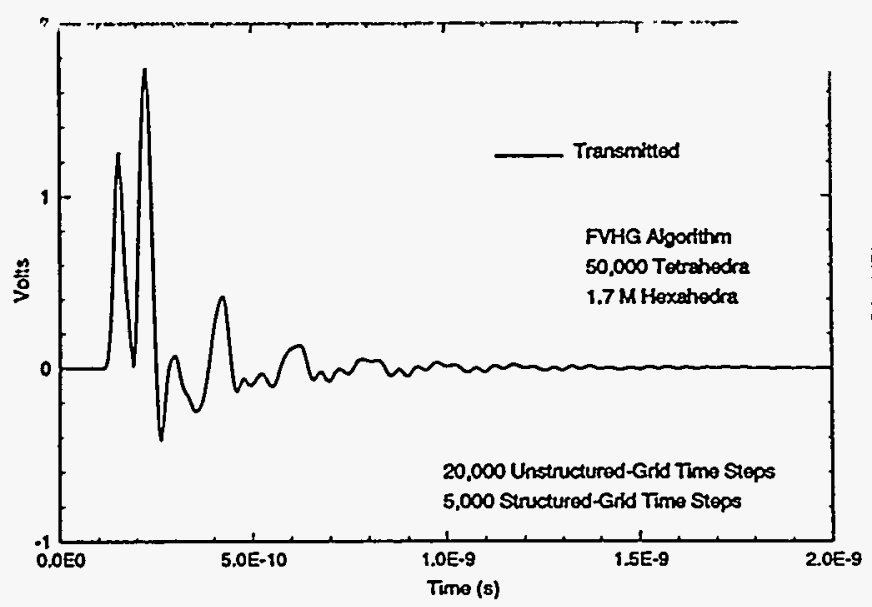

Fig. 3.7a. Time response at output observer for low-pass filter.

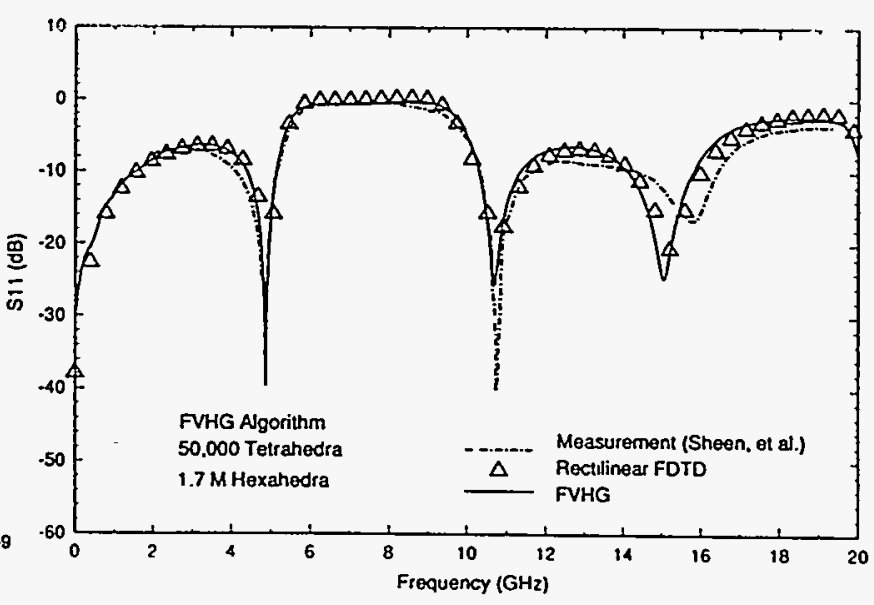

Fig. 3.7b. Return loss.

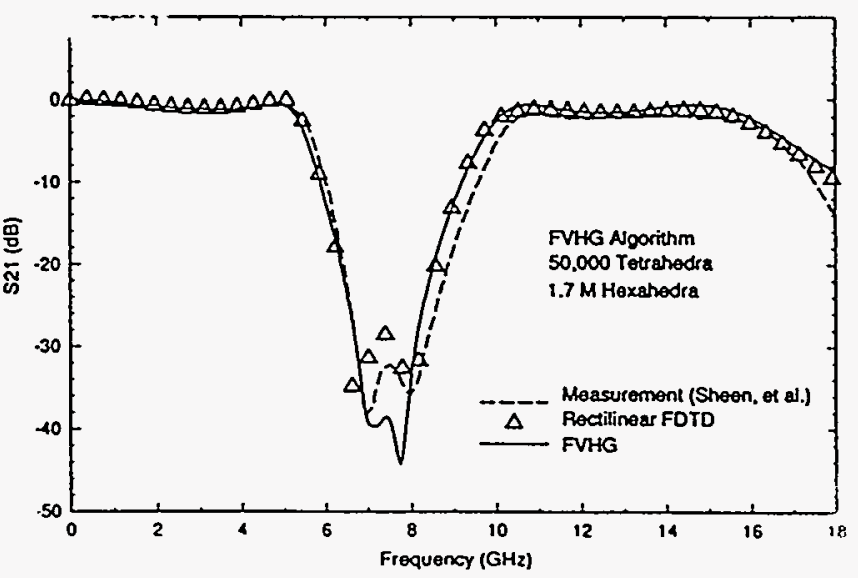

Fig. 3.8b. Insertion loss. 


\section{Microstrip Patch Antenna}

The final example is the well-known patch antenna originally studied by Sheen, et al., [22]. A portion of the antenna modeled here is shown in Fig. 3.9. For this problem, a low-density hexahedron grid, with an edge length equal to the substrate width $(0.794 \mathrm{~mm})$, was used with a highdensity tetrahedron grid of the geometry shown in Fig. 3.9. A surface grid with an average edge length of $0.3 \mathrm{~mm}$ was placed on the patch antenna. A portion of the unstructured grid showing the grid transition is shown in Fig. 3.10. The driving microstrip, including the source, observer, and termination planes, was in the low-density hexahedron region. The observer was located under the midpoint of the power trace and positioned 2 cells from the left edge of the grid shown in Fig. 3.10. A local first-order Mur boundary condition was applied at the left edge of this grid, and the source plane was located 15 cells from the left edge. Upon adding the wrapper, the un-

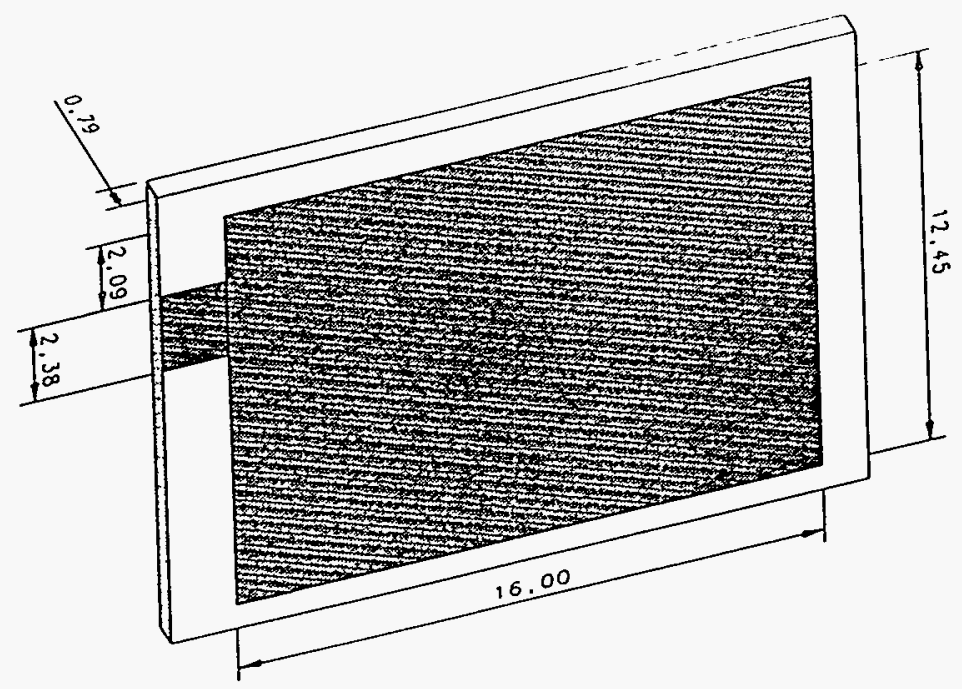

Fig. 3.9. M.jdel of patch antenna. Substrate relative permittivity is 2.2 . The input pori is at the trace "stub" on the left side of the figure. (Dimensions in $\mathrm{mm}$.)

structured grid was placed in a uniform FDTD grid that measured $96 \times 44 \times 60$. The FDTD grid used a uniform cell size of $0.794 \mathrm{~mm}$ and was terminated with second-order Mur boundaries. Note that the hexahedral grid only provides about 13 cells per wavelength in the substrate at 20 $\mathrm{GHz}$.

Using the high-density surface grid gave rise to a maximum to minimum edge length ratio on the primary grid of $11.4\left(R_{p}\right)$, while on the dual grid this ratio was $17.25\left(R_{d}\right)$. Twelve sub-time steps were required in the unstructured grid $\left(N_{\mathcal{U}}=12\right), N_{S}=2$, and the stabilization parameter, $\alpha$, was set to 3 (based on the guideline, $N_{S} \cdot N_{u} \cdot \alpha \leq 80$ ). The unstructured grid contained approximately 36,000 tetrahedra and 520 hexahedra (excluding the wrapper). Results at the input observer are shown in Figs. 3.11a,b. The simulation ran for 36,000 time steps in the 


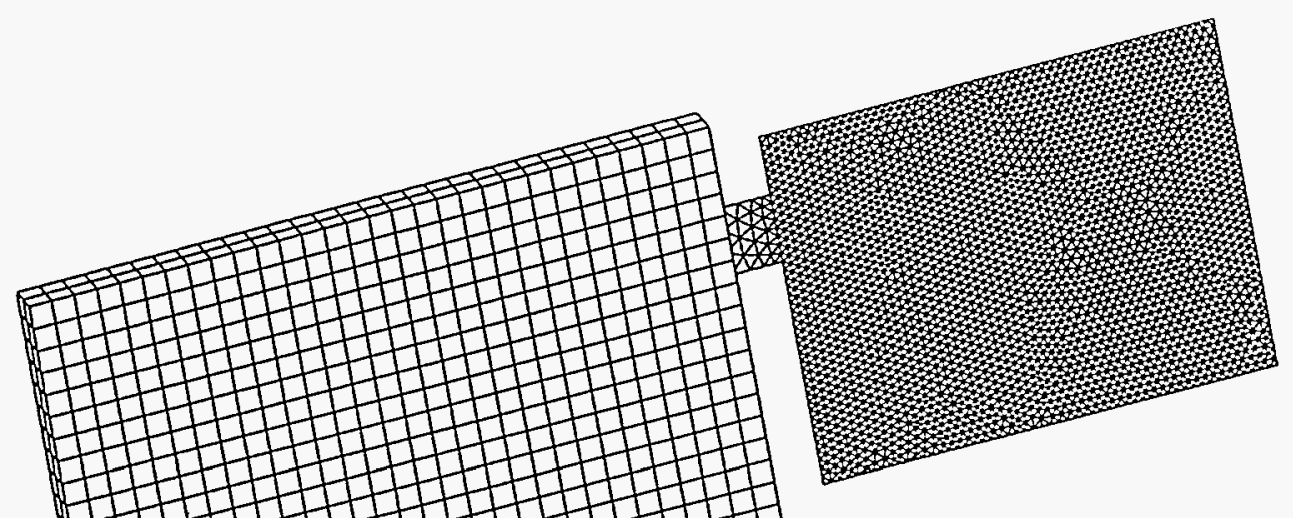

High density tetrahedrons

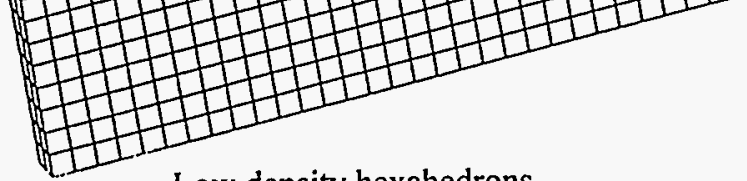

Low density hexahedrons

Fig. 3.10. Partial grid of patch antenna. The feeding trace, modeled with uniform quadrilateral shell elements, resides in the low-density hexahedron mesh and connects to the input port of the patch antenna. At this point, the grid transitions to a dense triangular-element surface mesh surrounded by tetrahedral elements.

unstructured grid (3,000 in the structured grid) and required approximately 6 hours using 6 processors of the Sun Sparcserver 1000. It is noted that the patch antenna required approximately 1 hour to generate the solid model, tetrahedra grid, and mapped-mesh hexahedra grid for the input trace extension using I-DEAS (cf. Fig. 3.10), as well as all primary and dual grid information from the preprocessor.

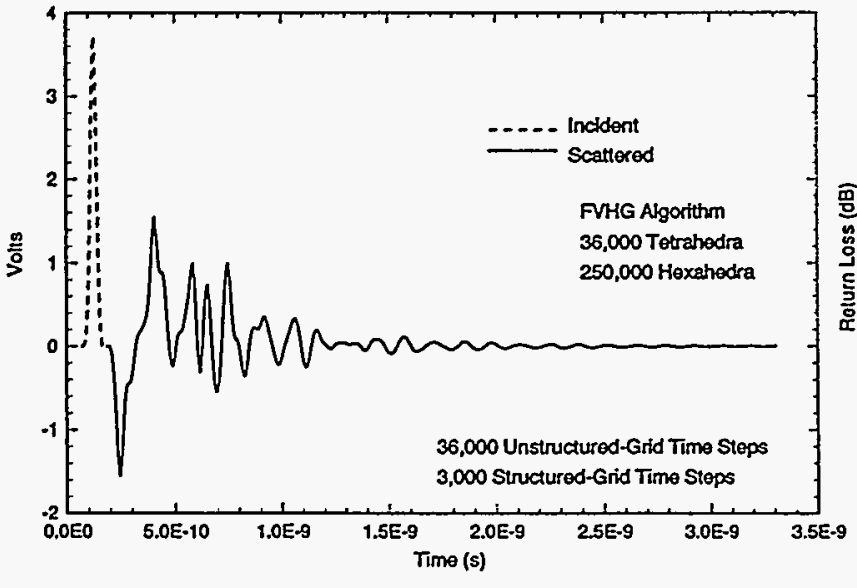

Fig. 3.1 1a. Time response at input observer for patch antenna.

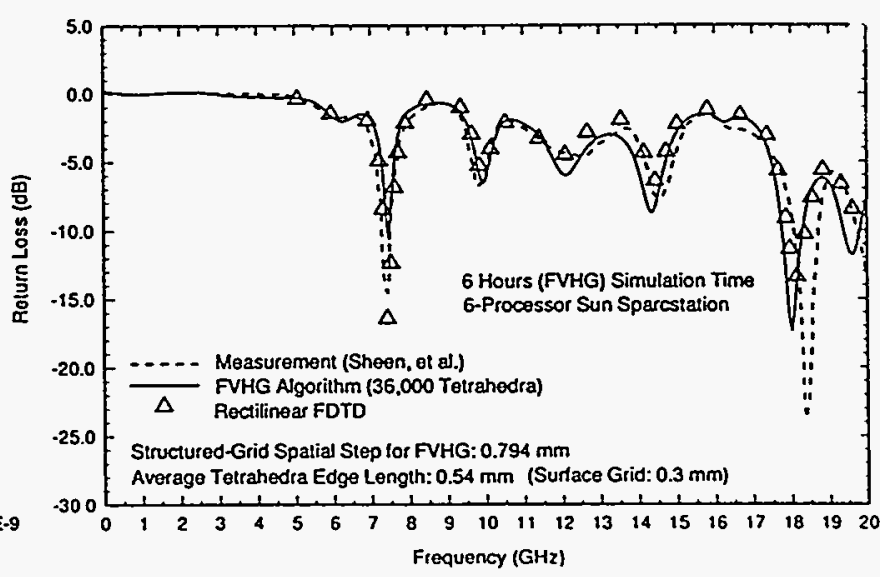

Fig. 3.11b. Return loss. 


\section{Conclusions}

This paper has examined the effect of local, free-meshed tetrahedron grids in uniform-grid FDTD as applied to microstrip geometries. By comparing with well known FDTD solutions, the FVHG approach was shown to provide very accurate results when specialized to rectangular geometries. Numerical reflections at the hexahedron-tetrahedron interfaces have typically been found to be suppressed by at least $40 \mathrm{~dB}$. Much of the reflection is believed to be due to current boundary condition approximations in the finite-volume formulation as applied to open conductors. Improved approximations remain an active topic of research. Abrupt changes in grid density and different dispersion characteristics between hexahedrons and tetrahedrons also contribute. However, the dynamic range that is currently obtainable is sufficient for most practical microstrip applications. Extensive numerical experimentation has revealed that a good indicator for the grid resolution of a tetrahedron primary grid is based on the maximum of the average edge lengths of the primary and dual grids. The upper frequency resolution is then obtained by equating this distance to the wavelength divided by twenty. Because the FVHG approach uses commercial solid-modeling and local free-meshed grids, the efficient modeling of high complexity devices is possible. Examples will be discussed in future papers.

The development and application of unstructured finite volume and finite element methods to transient computational electromagnetics is only beginning to evolve. Important research areas in FDTD such as radiation boundary conditions and sub-grid models will undoubtedly be revisited in this context. Additional research into the late-time stability of explicit difference schemes on complex grids is also needed. This paper has utilized an effective, variably dissipative timeaveraging scheme to obtain stability in the unstructured grid, but non-dissipative timedifferencing is ultimately desired.

\section{Acknowledgment}

The authors thank Dr. Mike Pasik for supplying comparison data for the microstrip low-pass filter and patch antenna obtained from the rectilinear FDTD code, QUICKSILVER [23].

\section{References}

[1] K.S. Yee, "Numerical solution of initial boundary value problems involving Maxwell's equations in isotropic media," IEEE Trans. Antennas Propagat., 14, 4, pp. 302-307, 1966.

[2] K.L. Shlager and J.B. Schneider, "A selective survey of the finite-difference time-domain literature," IEEE Antennas Propagat. Mag., 37, 4, pp.39-57, 1995.

[3] R. Holland, "Pitfalls of staircase meshing," IEEE Trans. EMC, 35, 4, pp.434-439, 1993.

[4] M.N.O. Sadiku, Numerical Techniques in Electromagnetics, pp. 407-500, CRC Press, FL, 1992.

[5] A. Chatterjee and J.L. Volakis, "Conformal absorbing boundary conditions for 3-D problems: derivation and applications," IEEE Trans. Antennas Propagat., 43, 8, pp. 860-866, 1994.

[6] C.H. Chan, J.T. Elson, and H. Sangani, "An explicit finite-difference time-domain method using Whitney elements," IEEE Antennas Propagat. Symp. Dig., 3, pp. 1768-1771, Seattle, WA, 1994.

[7] J.-F. Lee, "Whitney elements time domain (WETD) methods for solving three-dimensional waveguide discontinuities," IIth Annual Review of Progress in Applied Computational Electromagnetics (ACES) Symp. Dig., pp. 1258-1265, 1995.

[8] V. Shankar, A.H. Mohammadian, and W.F. Hall, "A time-domain, finite-volume treatment for the Maxwell equations," Electromagnetics, 10, 1-2, pp. 127-145, 1990. 
[9] N.K. Madsen and R.W. Ziolkowski, "A three-dimensional modified finite volume technique for Maxwell's equations," Electromagnetics, 10, 1-2, pp. 147-161, 1990.

[10] N.K. Madsen, "Divergence preserving discrete surface integral methods for Maxwell's curl equations using nonorthogonal unstructured grids," RIACS Tech. Rep. 92.04, NASA Ames Research Center, CA, 1992.

[11] K.S. Yee and J.S. Chen, "Conformal hybrid finite-difference time-domain (FDTD) with finite-volume time domain," IEEE Trans. Antennas Propagat., 42, 10, pp. 1450-1455, 1994.

[12] T.G. Jurgens and A. Taflove, "Three-dimensional contour FDTD modeling form single and multiple bodies," IEEE Trans. Antennas Propagat., 41, 12, pp. 1703-1708, 1993.

[13] D.J. Riley and C.D. Turner, "Unstructured finite-volume modeling in computational electromagnetics," 11th Annual Review of Progress in Applied Computational Electromagnetics (ACES) Symp. Dig., pp. 435-444, 1995.

[14] D.J. Riley and C.D. Turner, "Interfacing unstructured tetrahedron grids to structured-grid FDTD," IEEE Microwave and Guided Wave Letters, 5, 9, pp. 284-286, 1995.

[15] S.D. Gedney and F. Lansing, "A parallel discrete surface integral equation method for the analysis of three-dimensional microwave circuit devices with planar symmetry," IEEE Antennas Propagat. Symp. Dig., 3, pp. 1778-1781, Seattle, WA, 1994.

[16] CUBIT, Sandia National Laboratories, Albuquerque, NM, Dept. 1425, private communication, 1994.

[17] Hexagen, Algor, Inc., Pittsburgh, PA, 15238.

[18] T.J.R. Hughes, The Finite Element Method, pp. 490-569, Prentice-Hall, Englewood Cliffs, NJ, 1987.

[19] K.L. Shlager, J.G. Maloney, S.L. Ray, and A.F. Peterson, "Relative accuracy of several finite-difference time-domain methods in two and three dimensions," IEEE Trans. Antennas Propagat., 41, 12, pp. 1732-1737, 1993.

[20] W.F. Ames, Numerical Methods for Partial Differential Equations, pp.47-49, Academic Press, NY, NY, 1977.

[21] I-DEAS Master Series, Structural Dynamics Research Corporation, Milford, OH, 45150-2789.

[22] D.M. Sheen, S.M. Ali, M.D. Abouzahra, and J.A. Kong, "Application of the three-dimensional finite-difference time-domain method to the analysis of planar microstrip circuits," IEEE Trans. Microwave Theory and Tech., 38, 7, pp. 849-857, 1990.

[23] D.B. Seidel, M.L. Kiefer, R.S. Coats, T.D. Pointon, J.P. Quintenz, and W.A. Johnson, "The 3-D electromagnetic, particle-in-cell code, QUICKSILVER," Proceedings of the CP90 Europhysics Conference on Computational Physics, pp. 475-482, 1991. 


\section{Distribution}

Dr. N.K. Madsen

Lawrence Livermore National Laboratory

The University of California

Livermore, CA 94550

Dr. M.E. Jones

Los Alamos National Laboratory

The University of California

P.O. Box 1663

Low Alamos, NM 87545

Dr. K.S. Yee

Lockheed Martin Palo Alto Research Laboratory

Palo Alto, CA 94304

Dr. J. G. Maloney

Signature Technology Lab

Georgia Institute of Technology

Atlanta, GA 30332

Dr. K.K. Mei

Dept. of Electrical Engineering and Computer Science

Electronics Research Laboratory

The University of California

Berkeley, CA 94720

Dr. O.P. Gandhi

Dept. of Electrical Engineering

The University of Utah

Salt Lake City, UT 84112

Dr. J.L. Volakis

Radiation Laboratory

Dept. of Electrical Engineering and Computer Science The University of Michigan

Ann Arbor, MI 48109-2122

Dr. A.C. Cangellaris

Electromagnetics Laboratory

Dept. of Electrical and Computer Engineering

The University of Arizonia

Tucson, AZ 85721 


\section{Distribution (con't)}

Dr. R. Ziolkowski

Electromagnetics Laboratory

Dept. of Electrical and Computer Engineering

The University of Arizona

Tucson, AZ 85721

Dr. Cinzia Zuffada

Jet Propulsion Laboratory

California Institute of Technology

Pasadena, CA 91109

Dr. V.P. Cable

Lockheed Martin Advanced Development Company

P.O. Box 551

Burbank, CA 91520

Dr. A.C. Woo

NASA Ames Research Center

Moffett Field, CA 98124

Dr. R. J. Luebbers

Electrical Engineering Dept.

The Pennsylvania State University

University Park, PA 16802

Dr. Lyle Long

Dept. of Aerospace Engineering

The Pennsylvania State University

University Park, PA 16802

Dr. J-F Lee

ECE Dept.

WPI

Worcester, MA 01609

Dr. Vijaya Shankar

Rockwell International Science Center

Thousand Oaks, CA 91360 


\section{Distribution (con't)}

Dr. Steven P. Castillo

Department of Electrical and Computer Engineering

New Mexico State University

Department 3-O

Box 30001

Las Cruces, NM 88003

Mr. Jeff Frantz

Structural Dynamics Research Corporation

11995 El Camino Real, Suite 200

San Diego, CA 92130

Dr. Steven D. Gedney

Department of Electrical Engineering

University of Kentucky

Lexington, KY 40506

Dr. E.M. Buturla

Technology Computer Aided Design

SEMATECH

2706 Montopolis Dr.

Austin, TX 78741

Dr. Paul K.U. Wang

Pacific Numerix Corporation

7333 E. Doubletree Ranch Rd., Suite 280

Scottsdale, AZ 85258

$\begin{array}{lll}\text { MS } & 0533 & \text { Billy C. Brock } \\ \text { MS } & 1153 & \text { Larry D. Bacon } \\ \text { MS } & 1153 & \text { John F. Aurand } \\ \text { MS } & 0529 & \text { Richard T. Knudson } \\ \text { MS } & 1186 & \text { David B. Seidel } \\ \text { MS } & 1186 & \text { Michael F. Pasik } \\ \text { MS } & 1186 & \text { Mark L Kiefer } \\ \text { MS } & 0865 & \text { Marvin E. Morris } \\ \text { MS } & 0829 & \text { Russell D. Skocypec } \\ \text { MS } & 1166 & \text { C. David Turner } \\ \text { MS } & 1188 & \text { John S. Wagner } \\ \text { MS } & 9202 & \text { Rene Bierbaum } \\ \text { MS } & 0750 & \text { Gregory A. Newman } \\ \text { MS } & 0750 & \text { David L. Alumbaugh }\end{array}$




\section{Distribution (con't)}

MS 1165 James E. Powell

MS 1166 Gary J. Scrivner

MS 1190 Donald L. Cook

MS 1166 Douglas J. Riley (50)

MS 9018 Central Technical Files, 8523-2 (1)

MS 0899 Technical Library, 4414 (5)

MS 0619 Print Media, 12615 (1)

MS 0100 Document Processing, 7613-2, For DOE/OSTI (2) 